\title{
Which long-term illnesses do patients find most limiting? A census-based cross-sectional study of 340,000 people
}

\author{
David M. Wright $₫ \cdot$ Michael Rosato $\cdot$ Dermot O'Reilly
}

Received: 18 May 2016/Revised: 28 October 2016/Accepted: 17 November 2016/Published online: 9 December 2016 (C) The Author(s) 2016. This article is published with open access at Springerlink.com

\begin{abstract}
Objectives To investigate associations between a widely used measure of self-assessed health (limiting long-term illness, LLTI) and 11 long-term health conditions.

Methods Information on LLTI and health conditions was obtained from 2011 Census returns for a $28 \%$ representative sample of the Northern Ireland population $(n=342,868)$. Logistic regression was used to predict LLTI by sex and age group for each condition found in isolation, adjusting for marital status, social class, household car access, housing tenure, and educational attainment. The relationship between limitation and multimorbidity was also assessed.

Results Prevalence of LLTI varied considerably among conditions when found in isolation; those with mobility problems were over 50 times more likely to report limitation than those with hearing loss. Women were less likely to report limitation than men [OR $=0.93(0.90,0.96)]$, but the pattern of associations with health conditions was similar for both sexes. Prevalence of LLTI increased with age and number of health conditions.

Conclusions LLTI was most closely associated with mobility problems. Limitation increased slightly with age, but patterns of LLTI across conditions were not sex dependent.
\end{abstract}

Electronic supplementary material The online version of this article (doi:10.1007/s00038-016-0929-2) contains supplementary material, which is available to authorized users.

D. M. Wright ( $\square) \cdot$ D. O'Reilly

Centre for Public Health, Queen's University Belfast,

Belfast, UK

e-mail: d.wright@qub.ac.uk

M. Rosato

Bamford Centre for Mental Health and Wellbeing,

Ulster University, Londonderry, UK
Keywords Limiting long-term illness .

Self-assessed health · Census

\section{Introduction}

Census responses to questions on self-assessed health have played an important role in the assessment of health, need for health services, and the allocation of National Health Service resources in the United Kingdom for the past two decades. A question on long-term health conditions limiting daily activities (limiting long-term illnesses, LLTI) was introduced in the 1991 UK Census to provide a nationally consistent measure that would indicate healthcare need (Dale and Marsh 1993). Wording was adapted from the General Household Survey, where LLTI had been associated with both primary and secondary healthcare uses (Cohen et al. 1995; Dale and Marsh 1993). Aggregating Census returns by area, LLTI has been widely used to measure health inequalities (Barnett et al. 2001; Bentham et al. 1995; Shouls et al. 1996). LLTI has remained a key input variable to NHS resource allocation formulae for inpatient and General Practitioner services by small area (Rhys et al. 2010; Sheldon et al. 1994; Smith et al. 1994), so it is important to understand the characteristics of the measure.

An early investigation into the properties of the LLTI measure compared it with responses to a standard health survey for a sample of over 6000 people (Cohen et al. 1995). Those reporting LLTI also reported worse general and physical health; after adjusting for these factors, there was little association between LLTI and measures of mental health and social wellbeing. A similar design was used to estimate coincidence of LLTI and specific health 
conditions in a larger cohort (Payne and Saul 2000). LLTI was most closely correlated with the presence of physical conditions, especially angina or musculo-skeletal disease. In contrast to Cohen et al. (1995), poor mental health (depression) was found to be associated with LLTI. Subsequent mortality risk and likelihood of hospital admission were considerably higher for those reporting LLTI (Payne and Saul 2000). In both studies, rates of LLTI elicited from health surveys were higher than those from the Census indicating under-reporting of conditions in Census returns.

The framing and phrasing of the LLTI question have changed across Censuses, potentially revealing different aspects of self-assessed health. In the 2011 Census, the most important change was from a two category (yes/no) to a graded response to limitation: 'Are your day-to-day activities limited because of a health problem or disability which has lasted, or is expected to last, at least 12 months? (include problems related to old-age)', with three response options (Yes, limited a lot; Yes, limited a little; No). The three-category version has the potential to reveal more about the perceived limitation brought by different health conditions than the original and this wording is frequently used by employers for monitoring equality within the workplace. Although the 2011 question has been deemed 'broadly comparable' to the previous (2001) version by the Office for National Statistics (ONS 2012), to our knowledge, no assessment of how to interpret the new measure at the population scale has been attempted. We conducted such an assessment, addressing the broad question: when declaring their day-to-day activities to be limited a little or a lot, which chronic health conditions are people referring to?

We utilised responses to an additional health question asked in the Northern Ireland but not in the England and Wales Census: 'Do you have any of the following conditions which have lasted, or are expected to last, at least 12 months?'. Respondents could select either no condition or multiple conditions from a list of 11 (see Table 1; the Scottish Census asked the same stem question, but the list of conditions differed). Responses to the two questions were compared to generate a comprehensive picture of the associations between each of the health conditions and perceived limitation of daily activities. The list of health conditions contains a mixture of functional limitations, diseases, and symptoms, and some of which may be more commonly found in combination than in isolation. Symptoms are particularly likely to be found in combination with an underlying disease (e.g., long-term pain and chronic illness). Multimorbidity, defined as co-occurrence of two or more chronic medical conditions, is a growing public health problem in developed countries as prevalence and care costs increase with population aging (Barnett et al. 2012). Although limitation is likely to increase with the number of conditions, the form of this relationship is not well characterised at the population scale. Therefore, we considered associations between LLTI and each condition in isolation and in combination and also the association between LLTI and multimorbidity in general.

There is evidence that the relationship between selfassessed health and presence of underlying conditions differs between sexes (Mikolajczyk et al. 2008) and that men and women have different trajectories of self-assessed health as they age, associated with changes in employment or socio-economic status (SES) (Power et al. 2000; Rohlfsen and Kronenfeld 2014). In addition, prevalence of LLTI has been negatively associated with socio-economic status at both the individual and area levels (Boyle et al. 2004; Malmström et al. 2001; Zhang et al. 2013). Therefore, we also investigated whether the relationships between health conditions and self-assessed limitation of daily activities varied by age and sex whilst adjusting for variation in responses among socio-economic groups. Although there is some evidence that the assessment of health may vary among ethnic groups (Smith and Grundy 2011), it was not possible to explore this here given the ethnic homogeneity of the Northern Ireland population.

\section{Methods}

\section{Data source}

Cross-sectional data from the 2011 Northern Ireland Census were accessed through the Northern Ireland Longitudinal Study (NILS), a health card registrationbased linkage of Census records and vital events data for approximately $28 \%$ of the population (O'Reilly et al. 2012). The use of the NILS for research has been approved by Office for Research Ethics Committees Northern Ireland.

A total of 378,342 NILS members aged 16 and over were enumerated at the 2011 Census. Residents of communal establishments (4836) were excluded. A further 28,660 were excluded because responses to either the health conditions question or the LLTI question had been imputed due to non-response. Finally, another 1978 were excluded because answers to these questions were not required (e.g., returns at home addresses for non-resident students), leaving a cohort of 342,868 .

In addition to the demographic characteristics of age and sex, marital status, and religious affiliation [there is evidence that this may modify perception or reporting of selfreported health (O'Reilly and Rosato 2010)], the study also explored the relationship to measures of socio-economic status previously associated with variation in self-assessed health (Luchenski et al. 2008; Malmström et al. 2001; 
Table 1 Responses to 2011 Northern Ireland Census question 'Do you have any of the following conditions which have lasted, or are expected to last, at least 12 months?'

\begin{tabular}{ll}
\hline Condition definition & Abbreviation \\
\hline Deafness or partial hearing loss & Hearing loss \\
Blindness or partial sight loss & Sight loss \\
Communication difficulty (a difficulty with speaking or making yourself understood) & Communication \\
& difficulty \\
A mobility or dexterity disability (a condition that substantially limits one or more & Mobility difficulty \\
basic physical activities such as walking, climbing stairs, lifting or carrying) & \\
A learning difficulty, an intellectual difficulty, or a social or behavioural difficulty & Behavioural \\
& difficultly \\
An emotional, psychological or mental health condition (such as depression or & Mental health \\
schizophrenia) & condition \\
Long-term pain or discomfort & Long-term pain \\
Shortness of breath or difficulty breathing (such as asthma) & Breathing difficulty \\
Frequent periods of confusion or memory loss & Memory loss \\
A chronic illness (such as cancer, HIV, diabetes, heart disease or epilepsy) & Chronic illness \\
Other condition & Other condition \\
\hline
\end{tabular}

Murphy et al. 1997; O'Reilly and Rosato 2010). These were extracted from the Census and included social class as measured by the National Statistics Socio-economic Classification (NS-SEC) (Rose and Pevalin 2002), educational attainment, housing tenure, and car availability (see Table S1 for the categories of these variables).

In a general comparison against cohort members, a higher proportion of non-respondents were male, in lower socio-economic classes and living in rented accommodation (distributions not shown, available on request). Nonrespondents were more likely to be under 35 and much less likely to have stated a religion.

\section{Analytical approach}

The analysis was divided into two parts. First, we used a mixture of descriptive statistics and logistic regression to describe the associations between limitation of daily activities and health conditions. The analysis focused on estimates for the 11 health conditions when found in isolation to derive a clear picture of the relative influence of each condition independent of potential interactions due to multimorbidity (see Table 1 for variable descriptions). For each health condition, the modelling set consisted of those reporting either that condition or no health conditions (the reference group). Rather than directly fitting a multinomial model to the three-way classification of limitation, two binary response variables were derived (no limitation vs. any limitation; no limitation vs. limited a lot) and separate models fitted for each, estimating the probability of limitation among those with each health profile. This approach was chosen to allow comparison with previous studies that used the two-way classification of limitation (here represented by the no limitation vs. any limitation model) and to indicate whether inclusion of the third category gave additional insights (e.g., yielding substantially different estimates from the no limitation vs. limited a lot model). Models were adjusted for age (5 year age bands), sex, marital status, NS-SEC, educational attainment, religion, housing tenure, and car availability. The model was of the form:

$\operatorname{logit}(y)=\alpha+\beta_{1} x_{1}+\cdots+\beta_{p} x_{p}+\gamma_{j} c_{j}+\varepsilon$

(Model 1)

where the response $y$ was modelled by the intercept $\alpha$, and $\beta_{j} s$ were the coefficients of the covariates $x_{1}, \ldots, x_{p}$ representing the levels of the factors adjusted for $(p=27$ in the fully adjusted model; see Table $\mathrm{S} 1$ for full list of factor levels). $c_{j}$ was a dummy variable indicating the presence of the $j$ th condition ( $c_{j}=1$ if condition $j$ was present, $c_{j}=0$ if it was absent). $\gamma_{j}$ was the corresponding coefficient for the $j$ th condition, and $\varepsilon$ was the error term. The relationship between health profiles and limitation was also estimated for each sex and for different age groups by extending model 1 to include interactions. Three broad age bands were chosen to represent different major life stages: youth $(<25)$, adulthood (25-65), and retirement ( $>65)$. Probability of reporting limitation was predicted for each age-sex group marginalising over the covariates using the effects package in the $R$ software environment (R Development Core Team 2015).

Second, we investigated the relationship between limitation and multimorbidity. We estimated the association between number of health conditions and probability of reporting each level of limitation, adjusting for covariates. In model 2 , the $\gamma_{j} c_{j}$ term in model 1 was replaced with $\delta_{1} d_{1}+\cdots+\delta_{5} d_{5}$, where $d_{1}, \ldots, d_{5}$ were dummy variables indicating the number of reported conditions. For a single reported condition, $d_{1}=1$ and $d_{2}, \ldots, d_{5}$ all zero; for two conditions, $d_{1}=0, d_{2}=1, d_{3}, \ldots, d_{5}$ all zero. Five or more conditions were denoted by $d_{5}=1$ and $d_{1}, \ldots, d_{4}$ all zero. 
$\delta_{1} \ldots \delta_{5}$ were coefficients corresponding to the number of reported conditions.

Previous studies have indicated that mobility-related conditions are likely to be strongly associated with limitation, so the analysis was repeated excluding records for those with mobility problems. We also estimated the relative influence of each health condition adjusting for the influence of covariates and other conditions by fitting multiple regressions to the full data set (i.e., including both isolated cases and those with multimorbidity) with dummy variables for each of the 11 conditions $c_{1}$ to $c_{11}$ :

$\log i t(y)=\alpha+\beta_{1} x_{1}+\cdots+\beta_{p} x_{p}+\gamma_{1} c_{1}+\cdots+\gamma_{11} c_{11}+\varepsilon$.

(Model 3)

\section{Results}

Degree of limitation

Overall, 48,186 people (14.1\%) reported that daily activities were limited a lot as a result of long-term health conditions; a further 36,422 (10.6\%) reported a little limitation. Prevalence of limitation (especially 'limited a lot') increased with age (Fig. S1). Prevalence of health conditions is shown in Table 2. The most prevalent (each $>9 \%$ ) was mobility problems, pain, and breathing difficulties. Least prevalent (each $<2 \%$ ) was blindness, behavioural, or communication difficulties. For each condition, the majority of cases were found in combination with other conditions. The proportion of isolated cases ranged from $5.4 \%$ (communication difficulties) to almost half ('other conditions').

Health conditions found in isolation varied considerably in perceived limitation of daily activities. The odds ratios in Table 3 are relative to those with no health conditions and so are understandably large, but health conditions can be compared with each other by division and comparison of confidence intervals. Mobility problems were most strongly associated with limitation with adjusted odds of reporting any limitation over 50 times greater than for those with hearing loss, the most weakly associated condition. Those with long-term pain, behavioural conditions, memory loss, or mental health problems were approximately eight times as likely to report limitation as those with hearing loss.

Among those reporting limitation, the proportion limited a lot also varied among conditions. Odds of reporting any limitation were similar for those with chronic illness or sight loss, but odds of reporting a lot of limitation were $40 \%$ lower for those with chronic illness. The pattern among conditions for those with a lot of limitation was similar to that for any limitation: those with mobility problems and hearing loss were most and least likely to report a lot of limitation, respectively (Table 3). There were a few anomalies: long-term pain was strongly associated with any limitation, but the ranking for a lot of limitation was lower, indicating that a large proportion of respondents reported that pain limited daily activities only a little (Table 3). Behavioural and 'other' conditions showed similar changes in rank between degrees of limitation.

Model specificity was high for both models (any limitation $=0.97$; limited a lot $=0.99$ ), but sensitivity was low (any limitation $=0.58$; limited a lot $=0.19$ ) indicating that prevalence of limitation was underestimated when conditions were considered in isolation.

Age, sex, and degree of limitation

Fully adjusted models indicated that females were less likely to report limitation than males (ORs 0.93 and 0.81 for any limitation and a lot of limitation, respectively), but the pattern of limitation across health conditions was similar across sexes (e.g., mobility problems were the most strongly associated with limitation for both sexes-not shown). Furthermore, relationships between health conditions and age did not differ substantially between sexes, and therefore, results from a model adjusting for sex (but with no age-sex-condition interactions) are reported.

Relationships between isolated health conditions, age, and limitation varied slightly among conditions. Inclusion of interactions between age group (three-way classification) and condition significantly improved model fit (likelihood ratio test, deviance $=1837, d f=71$, $P<0.001)$. For the majority of conditions, there were moderate increases with age in the probability of reporting any limitation (Fig. 1). Probability of limitation increased by $18 \%$ from the youngest to oldest age groups for those reporting a mental health condition. The greatest increases with age were between the adult and oldest age group for those with breathing or communication difficulties (30 and $45 \%$ increases, respectively).

A similar pattern was evident where daily activities were limited a lot, but increases were more subtle (Fig. 1). Those with breathing difficulties reported significantly increased risk of a lot of limitation from the adult to the oldest age group, but the difference was only $10 \%$. Although the point prediction for a lot of limitation among those in the youngest age group with memory loss was high, there was considerable uncertainty around the prediction due to the small size of the group, and the difference between age groups was not statistically significant.

With the exception of breathing and communication difficulties, the pattern of relative risks among conditions 
Table 2 Prevalence of health conditions both found in isolation and in combination with other conditions for the Northern Ireland 2011 Census, population aged $16+$

\begin{tabular}{|c|c|c|c|c|c|}
\hline \multirow[t]{2}{*}{ Health condition } & \multicolumn{2}{|l|}{ Prevalence $(\%)$} & \multirow[t]{2}{*}{ Not limited } & \multirow[t]{2}{*}{ Limited a little } & \multirow[t]{2}{*}{ Limited a lot } \\
\hline & Isolated condition & With other conditions & & & \\
\hline Mobility difficulty & 2.3 & 11.9 & 7.6 & 47.2 & 45.2 \\
\hline Behavioural difficulty & 0.4 & 1.1 & 48.8 & 29.0 & 22.2 \\
\hline Memory loss & 0.1 & 1.9 & 22.5 & 39.3 & 38.2 \\
\hline Long-term pain & 2.4 & 10.7 & 42.3 & 41.8 & 15.8 \\
\hline Mental health condition & 2.5 & 4.5 & 46.9 & 27.3 & 25.8 \\
\hline Communication difficulty & 0.1 & 1.2 & 52.7 & 25.3 & 21.9 \\
\hline Other condition & 2.7 & 3.3 & 60.5 & 30.8 & 8.7 \\
\hline Chronic illness & 3.0 & 5.3 & 56.2 & 31.1 & 12.7 \\
\hline Sight loss & 0.4 & 1.5 & 52.6 & 26.4 & 20.9 \\
\hline Breathing difficulty & 3.5 & 6.0 & 79.5 & 15.9 & 4.6 \\
\hline Hearing loss & 2.1 & 4.3 & 78.8 & 16.6 & 4.6 \\
\hline No conditions & 63.5 & - & 99.0 & 0.9 & 0.1 \\
\hline
\end{tabular}

Distribution of limitation among those with health conditions found in isolation (row percentages). Rows are ordered by the probability of reporting any limitation

Table 3 Estimated odds ratios (ORs) of reporting any or a lot of limitation by health condition for the Northern Ireland 2011 Census, population aged $16+$

\begin{tabular}{|c|c|c|c|c|c|c|}
\hline \multirow[t]{2}{*}{ Health condition } & \multicolumn{2}{|l|}{ In isolation } & \multicolumn{2}{|c|}{ Adjusting for other conditions } & \multicolumn{2}{|c|}{ Ranking (any limitation) } \\
\hline & Any limitation & Limited a lot & Any limitation & Limited a lot & In isolation & $\begin{array}{l}\text { Adjusting for } \\
\text { other conditions }\end{array}$ \\
\hline Mobility difficulty & $755(686,831)$ & $336(298,379)$ & $123(116,131)$ & $19.0(18.3,19.8)$ & 1 & 1 \\
\hline Behavioural difficulty & $110(97.6,124)$ & $154(130,183)$ & $52.8(47.0,59.2)$ & $21.0(18.2,24.1)$ & 2 & 2 \\
\hline Memory loss & $109(85.8,138)$ & $194(155,243)$ & $47.9(37.8,60.6)$ & $19.4(15.8,23.7)$ & 3 & 3 \\
\hline Long-term pain & $108(101,115)$ & $90.3(79.6,102)$ & $23.1(22.0,24.2)$ & $4.3(4.1,4.5)$ & 4 & 6 \\
\hline Mental health condition & $107(100,114)$ & $176(156,243)$ & $38.2(36.3,40.2)$ & $13.5(12.7,14.3)$ & 5 & 4 \\
\hline Communication difficulty & $69.4(52.6,91.6)$ & $127(90.5,178)$ & $31.5(23.9,41.6)$ & $15.0(10.8,20.9)$ & 6 & 5 \\
\hline Other condition & $56.9(53.5,60.5)$ & $51.0(44.8,58.2)$ & $21.4(20.4,22.5)$ & $4.9(4.6,5.2)$ & 7 & 8 \\
\hline Chronic illness & $52.8(49.7,56.1)$ & $69.2(61.1,78.5)$ & $17.9(17.1,18.8)$ & $4.7(4.4,4.9)$ & 8 & 9 \\
\hline Sight loss & $52.0(45.9,58.8)$ & $114(95.9,136)$ & $23.1(20.5,26.0)$ & $12.4(10.8,14.3)$ & 9 & 7 \\
\hline Breathing difficulty & $24.0(22.5,25.5)$ & $28.5(24.8,32.8)$ & $6.2(5.9,6.6)$ & $2.2(2.1,2.4)$ & 10 & 10 \\
\hline Hearing loss & $13.3(12.3,14.4)$ & $21.6(18.5,25.3)$ & $3.6(3.4,3.8)$ & $1.4(1.3,1.5)$ & 11 & 11 \\
\hline
\end{tabular}

Reference group—no health conditions. ORs and 95\% confidence intervals given for conditions found in isolation (Model 1) and when adjusting for the influence of the other health conditions (see equation-Model 3). All models adjusted for age, sex, education, religion, marital status, social class, car access, and housing tenure. Estimates for covariates from model 3 are reported in Table S1. Conditions are ranked by decreasing probability of reporting any limitation

varied little with age (the probability of reporting any limitation was greatest for those with mobility problems and least for those with hearing loss in all age groups).

\section{Limitation and multimorbidity}

The majority $(63.5 \%)$ of respondents reported no long-term health conditions. A slightly greater proportion reported isolated rather than two or more conditions (19.5 and
$17.0 \%$, respectively). Prevalence of multimorbidity increased with age; $10 \%$ of those aged 70 and above had four or more conditions (Fig. S2).

Limitation of daily activities increased with number of health conditions; predicted probability of reporting a lot of limitation increased from $13 \%$ (single condition) to $84 \%$ (five or more conditions-Fig. 2). A very small (1\%) proportion reported limitation without reporting a longterm condition. Among those with health conditions, the 
Fig. 1 Degree of limitation of day-to-day activities predicted for people with isolated longterm health conditions by age group, Northern Ireland 2011 Census, population aged $16+$. Predictions were made with covariates set at typical values (i.e., representing distribution within the population)

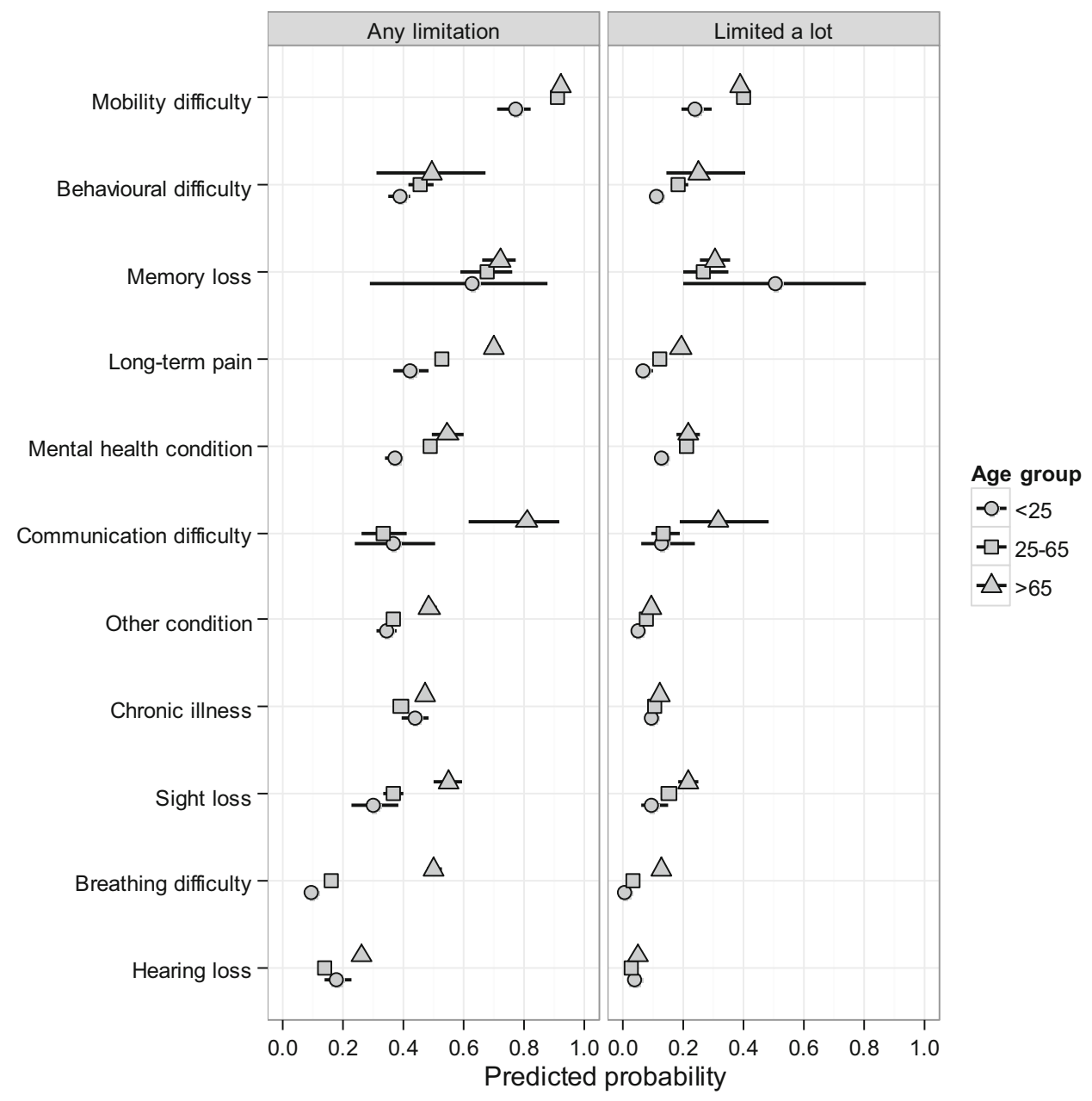

increase in proportion reporting any limitation was greatest from none to one condition (1-40\%), and from two conditions up, the majority reported a lot of limitation. When those with mobility problems were excluded, the probability of reporting either any or a lot of limitation decreased considerably, regardless of the number of other conditions (Fig. 2).

Associations between health conditions and limitation adjusting for the influence of other conditions were between one-sixth and half the size of estimates for conditions found in isolation (Table 3). However, the pattern of associations found in both analyses was similar. Ranking of most conditions was unchanged indicating that their relative influence was similar in both single and multimorbid cases (Table 3). Mobility problems and hearing loss were again the most strongly and weakly associated with limitation, respectively. There were a few differences between the sets of estimates. For any limitation, long-term pain was more highly ranked in isolation (4/11) than within the entire data set $(6 / 11)$, indicating that when found with other conditions, it is perceived to have a relatively minor influence on limitation. Conversely, sight loss was ranked more highly in the entire data set (7/11) than in isolation (9/11). These differences were more pronounced when modelling a lot of limitation. Model sensitivity of adjusted models was similar to unadjusted models (any limitation $=0.91$, limited a lot $=0.98$ ). Although still conservative, specificity was improved considerably following adjustment (any limitation $=0.71$, limited a lot $=0.55$ ).

\section{Discussion}

Using a large sample drawn from the 2011 Northern Ireland Census, we conducted a comprehensive assessment of the associations between long-term health conditions and self-assessed limitation of daily activities, and how these perceptions varied with age and sex.

Mobility problems were most prevalent and were most strongly associated with a lot of limitation of daily activities and in multimorbid cases, mobility problems had a disproportionately large influence on the presence and degree of limitation. These findings were consistent with a study in Great Britain using the two-category classification of long- 


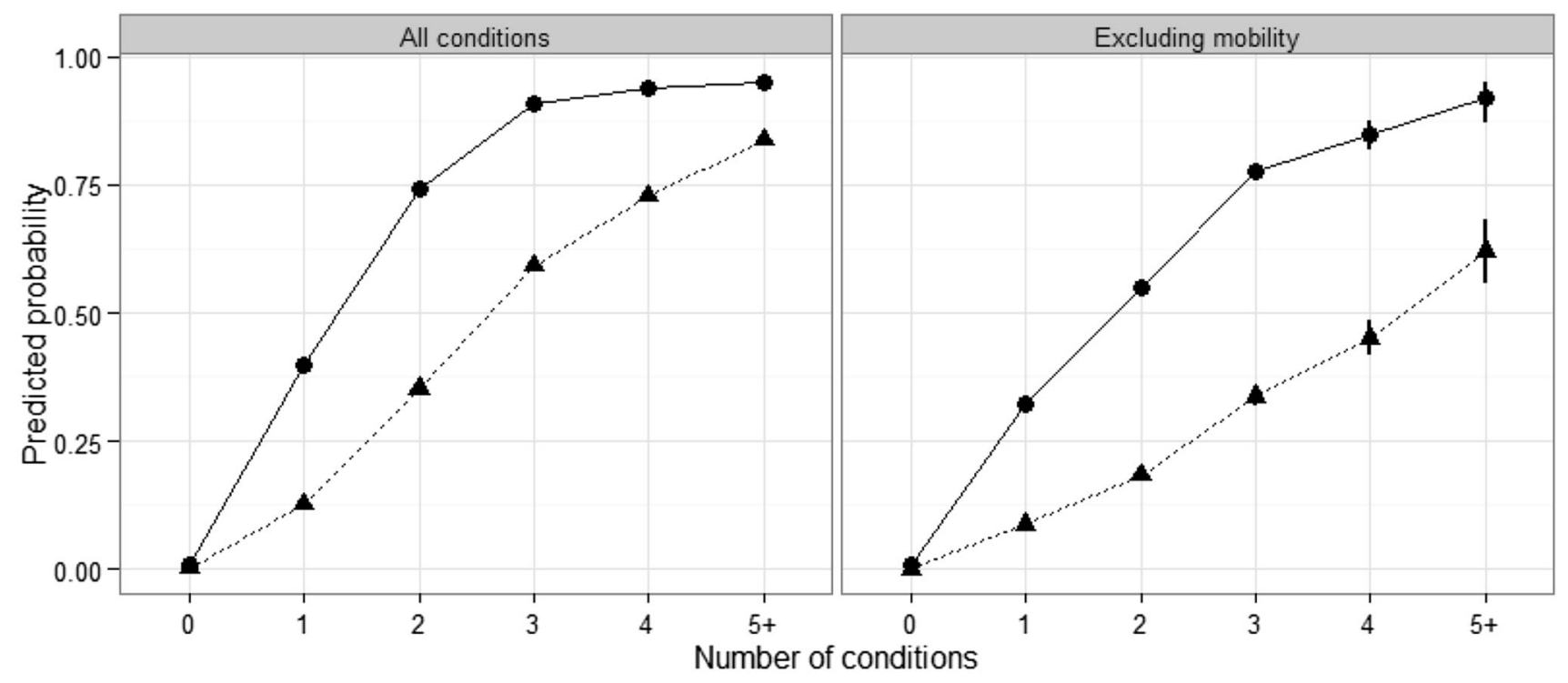

Limitation $\rightarrow$ Any limitation $-\mathbf{L}$ Limited a lot

Fig. 2 Degree of limitation of day-to-day activities by number of health conditions, Northern Ireland 2011 Census, population aged 16+. Estimates are from the entire data set (left hand panel) and a data set excluding all cases with mobility problems (right-hand

term limiting illness, where the strongest associations were with symptoms of musculo-skeletal problems, angina, or depression (Payne and Saul 2000). In contrast, chronic pain also had high prevalence, but limitation was slight in the majority of cases and the influence of pain was eclipsed by that of other conditions in multimorbid cases. Chronic pain and mobility have also been shown to profoundly affect self-assessed general health, second only to vitality in terms of influence (Au and Johnston 2014). Mental health problems and breathing difficulties were also reasonably prevalent, but breathing difficulties were associated with less limitation of daily activities, perhaps due to the episodic nature and good response to treatment of asthma and similar conditions. The lack of limitation associated with hearing loss may indicate that the majority of people have successfully adapted to the condition (e.g., through use of hearing aids) or that only one ear is affected (the same may apply for sight loss, which may be uni- or bilateral).

The overall prevalence of health conditions and limitation increased considerably with age, but associations between most health conditions and perceived limitation strengthened only slightly, with the exception of breathing or communication difficulties which showed considerable increases in the prevalence of limitation (albeit mild) past the age of 65 . Communication difficulties may be particularly limiting in old age as they may hinder access to health and social services, of particular importance among those living alone. However, in the older age group, those reporting isolated rather than multiple conditions are panel). Predictions were made with covariates set at typical values (i.e., representing distribution within the population). Sample sizes (entire data set): no conditions $=217,811$; one 66,919, two 25,165, three 16,584, four 9198, five plus 7191

relatively unusual (across all age groups, only $5 \%$ of cases of communication difficulty were reported in isolation), and so drawing strong inferences from this subgroup would be inappropriate. Breathing difficulties were much more prevalent (singly) in the oldest group, so respiratory conditions common in this group (e.g., chronic lung damage) appear to have a disproportionately large impact on functional status (i.e., avoiding disability and dependency) than more manageable conditions common among the young (e.g., asthma) (Ho et al. 2001; Nejjari et al. 1994).

The small disadvantage that we observed for men is consistent with reports of more LLTI and poorer general health among men than women across Ireland (O'Reilly et al. 2006). However, relative self-assessed health profiles of men and women vary considerably among studies and over time (Barnett et al. 2001; Power et al. 2000; Rohlfsen and Kronenfeld 2014; Young et al. 2010). A review of gender inequalities in self-assessed health found no consistent pattern of advantage for either sex across 48 countries (Beckfield et al. 2013). Health advantages of one sex in a given area may disappear or be reversed in a neighbouring area, with geographical variation partially associated with differential exposure to occupational health hazards (Senior 1998).

This study is one of few to have investigated whether the sexes responses to existing conditions vary in terms of selfassessed health (i.e., whether there is differential vulnerability). In a comparison of the relative influence of the two processes in an aging population, Rohlfsen and Kronenfeld 
(2014) found that faster declines in self-assessed health among men than women were associated with greater exposure to detrimental changes in social and health status. Differential vulnerability to these and other factors played a much less significant role. Similarly, we found that associations between a range of health conditions and limitation were similar for both sexes, so sex differences in self-assessed health are probably the result of differential exposure to risk factors rather than differential vulnerability. We used the recently introduced three category (not limited/limited a little/limited a lot) LLTI classification which gave additional insight into the relative influence of health conditions on daily activities, compared with studies using the two-category (not limited/limited) classification. Inclusion of the 'limited a lot' category enabled us to rank conditions which had similar limitation profiles under the two-category classification (e.g., sight loss and chronic illness). Three-category analysis clarified the relationship between number of health conditions and degree of limitation. Isolated conditions were most likely to be associated with a little limitation but limitation increased dramatically (and the majority were limited a lot) with two or more conditions, especially if mobility problems were present.

Our study had limitations. Census response rates are not uniform across demographic groups, with young adult males less likely to respond than others (NISRA 2015), potentially biasing estimates of limitation in the youngest age group. Non-respondents removed from our cohort tended to be in lower socio-economic classes and hence perhaps more likely to have health conditions than cohort members. However, even for these groups, Census sample size and response rates exceeded those of many surveys and as the overall prevalence of limitation among young adults was very low, a large disparity in limitation between non-respondents and cohort members would have been necessary to introduce substantial bias into overall estimates. More problematic for estimating the relative limitation of health condition was potential under-reporting of particular conditions due to social stigma. In Northern Ireland, this is most likely to have occurred among those with mental health problems, for which considerable stigma exists (c.f. stigma in the Republic of IrelandO'Keeffe et al. 2015). Under-reporting may have reduced the estimated prevalence of mental health problems and the proportion of those reporting a lot of limitation. There is ongoing work to assess the relative stigma associated with each condition by comparing the characteristics of those reporting each condition and those receiving treatment for it. Prevalence of all conditions may have been underestimated, because levels of self-assessed limitation within a population are typically higher under a targeted investigation of health status in comparison with Census responses (Cohen et al. 1995; Payne and Saul 2000).
Under-reporting may also be more prominent among those with poor educational attainment (Mackenbach et al. 1996). Finally, as this was a cross-sectional study, it was not possible to separate age and cohort effects or to infer whether observed associations between health conditions and limitation were causal.

A strength of this study was that information on health conditions and LLTI was obtained simultaneously, eliminating the possibility of misclassification due to status changes between collection of each type of information (e.g., development of a new health condition after limitation, but before health conditions had been assessed). In addition, because all responses were from the same source, potential biases introduced by difficulties linking Census with other data sets have been avoided (O'Reilly et al. 2008). Furthermore, the ordering of the two questions in the Census (LLTI first, health conditions second) suited our purposes, reducing the probability that responses to the limitation question was 'prompted' by the health conditions question as the context in which the LLTI question is asked can influence responses (Cohen et al. 1995; Payne and Saul 2000).

\section{Conclusions}

Mobility problems were identified from a range of 11 health conditions as most closely associated with selfassessed limitation of daily activities. Perceived limitation increased slightly with age, but patterns of perceived limitation across conditions were not sex dependent.

Acknowledgements The help provided by the staff of the Northern Ireland Longitudinal Study (NILS) and the NILS Research Support Unit is acknowledged. The NILS is funded by the Health and Social Care Research and Development Division of the Public Health Agency (HSC R\&D Division) and NISRA. The NILS-RSU is funded by the ESRC and the Northern Ireland Government. The authors alone are responsible for the interpretation of the data and any views or opinions presented are solely those of the authors and do not necessarily represent those of NISRA/NILS.

Ethical approval All procedures performed in studies involving human participants were in accordance with the ethical standards of the institutional and/or national research committee and with the 1964 Helsinki declaration and its later amendments or comparable ethical standards. For this type of study, formal consent is not required.

Funding This study was funded by the Economic and Social Research Council Grant ES/L007509/1 and the Northern Ireland Public Health Agency-Health and Social Care R\&D division Grant STL/4918/13.

Open Access This article is distributed under the terms of the Creative Commons Attribution 4.0 International License (http:// creativecommons.org/licenses/by/4.0/), which permits unrestricted use, distribution, and reproduction in any medium, provided you give appropriate credit to the original author(s) and the source, provide a link to the Creative Commons license, and indicate if changes were made. 


\section{References}

Au N, Johnston DW (2014) Self-assessed health: what does it mean and what does it hide? Soc Sci Med. doi:10.1016/j.socscimed. 2014.10.007

Barnett S, Roderick P, Martin D, Diamond I (2001) A multilevel analysis of the effects of rurality and social deprivation on premature limiting long term illness. J Epidemiol Comm Health. doi:10.1136/jech.55.1.44

Barnett K, Mercer SW, Norbury M, Watt G, Wyke S, Guthrie B (2012) Epidemiology of multimorbidity and implications for health care, research, and medical education: a cross-sectional study. The Lancet. doi:10.1016/S0140-6736(12)60240-2

Beckfield J, Olafsdottir S, Bakhtiari E (2013) Health Inequalities in Global Context. Am Behav Scient. doi:10.1177/0002764213487 343

Bentham G, Eimermann J, Haynes R, Lovett A, Brainard J (1995) Limiting long-term illness and its associations with mortality and indicators of social deprivation. J Epidemiol Comm Health. doi:10.1136/jech.49.Suppl_2.S57

Boyle P, Norman P, Rees P (2004) Changing places. Do changes in the relative deprivation of areas influence limiting long-term illness and mortality among non-migrant people living in nondeprived households? Soc Sci. doi:10.1016/j.socscimed.2003.09. 011

Cohen G, Forbes J, Garraway M (1995) Interpreting self reported limiting long term illness. BMJ. doi:10.1136/bmj.311.7007.722

Dale A, Marsh C (1993) The 1991 Census User's Guide. HMSO, London

Ho SF, O'Mahony MS, Steward JA, Breay P, Buchalter M, Burr ML (2001) Dyspnoea and quality of life in older people at home. Age Ageing. doi:10.1093/ageing/30.2.155

Luchenski S, Quesnel-Vallée A, Lynch J (2008) Differences between women's and men's socioeconomic inequalities in health: longitudinal analysis of the Canadian population, 1994-2003. J Epidemiol Comm Health. doi:10.1136/jech.2007.068908

Mackenbach JP, Looman CW, van der Meer JB (1996) Differences in the misreporting of chronic conditions, by level of education: the effect on inequalities in prevalence rates. Am J Public Health. doi:10.2105/AJPH.86.5.706

Malmström M, Johansson S, Sundquist J (2001) A hierarchical analysis of long-term illness and mortality in socially deprived areas. Soc Sci Med. doi:10.1016/S0277-9536(00)00291-4

Mikolajczyk R, Brzoska P, Maier C, Ottova V, Meier S, Dudziak U, Ilieva S, El Ansari W (2008) Factors associated with self-rated health status in university students: a cross-sectional study in three European countries. BMC Public Health

Murphy M, Glaser K, Grundy E (1997) Marital Status and Long-Term Illness in Great Britain. J Marriage Family

Nejjari C, Tessier J, Barberger-Gateau P, Jacqmin H, Dartigues J, Salamon R (1994) Functional status of elderly people treated for asthma-related symptoms: a population based case-control study. Euro Respirat J

NISRA (2015) Northern Ireland Census 2011 Quality assurance report

O'Keeffe D, Turner N, Foley S, Lawlor E, Kinsella A, O'Callaghan E, Clarke M (2015) The relationship between mental health literacy regarding schizophrenia and psychiatric stigma in the
Republic of Ireland. J Mental Health. doi:10.3109/09638237. 2015.1057327

O'Reilly D, Rosato M (2010) Dissonances in self-reported health and mortality across denominational groups in Northern Ireland. Soc Sci Med. doi:10.1016/j.socscimed.2010.05.042

O'Reilly D, Rosato M, Catney G, Johnston F, Brolly M (2012) Cohort description: the Northern Ireland Longitudinal Study (NILS). Int J Epidemiol. doi:10.1093/ije/dyq271

ONS (2012) 2011-2001 Census in England and Wales questionnaire comparability

O'Reilly D, Thompson KJ, Murphy AW, Bury G, Gilliland A, Kelly A, O'Dowd T, Steele K (2006) Socio-economic gradients in selfreported health in Ireland and Northern Ireland. Ir J Med Sci. doi:10.1007/BF03169172

O'Reilly D, Rosato M, Connolly S (2008) Unlinked vital events in census-based longitudinal studies can bias subsequent analysis. J Clin Epidemiol. doi:10.1016/j.jclinepi.2007.05.012

Payne N, Saul C (2000) What common disorders do those reporting limiting long-term illness experience, and what is their survival and health service utilization experience? J Public Health. doi:10.1093/pubmed/22.3.324

Power C, Li L, Manor O (2000) A prospective study of limiting longstanding illness in early adulthood. Int J Epidemiol. doi:10. 1093/ije/29.1.131

R Development Core Team (2015) R: A language and environment for statistical computing. R Found Stat Comput, Vienna

Rhys G, Beerstecher HJ, Morgan CL (2010) Primary care capitation payments in the UK. An observational study. BMC Health Services Res. doi:10.1186/1472-6963-10-156

Rohlfsen LS, Kronenfeld JJ (2014) Gender differences in trajectories of self-rated health in middle and old age: an examination of differential exposure and differential vulnerability. J Aging Health. doi:10.1177/0898264314527477

Rose D, Pevalin D (2002) A Researcher's guide to the national statistics socio- economic classification. Sage, London

Senior ML (1998) Area variations in self-perceived limiting long term illness in Britain, 1991: is the welsh experience exceptional? Reg Stud. doi:10.1080/713693455

Sheldon TA, Smith P, Borowitz M, Martin S, Hill RC (1994) Attempt at deriving a formula for setting general practitioner fundholding budgets. BMJ. doi:10.1136/bmj.309.6961.1059

Shouls S, Congdon P, Curtis S (1996) Modelling inequality in reported long term illness in the UK: combining individual and area characteristics. J Epidemiol Comm Health. doi:10.1136/ jech.50.3.366

Smith NR, Grundy E (2011) Time period trends in ethnic inequalities in limiting long term illness in England and Wales. Ethn Inequal Health Social Care. doi:10.1108/17570981111250859

Smith P, Sheldon TA, Carr-Hill R, Martin S, Peacock S, Hardman G (1994) Allocating resources to health authorities: results and policy implications of small area analysis of use of inpatient services. BMJ: Br Med J

Young H, Grundy E, O'Reilly D, Boyle P (2010) Self-rated health and mortality in the UK: results from the first comparative analysis of the England and Wales, Scotland, and Northern Ireland Longitudinal Studies. Popul Trends

Zhang X, Cook P, Lisboa P, Jarman I, Bellis M (2013) The effects of deprivation and relative deprivation on self-reported morbidity in England: an area-level ecological study. Int J Health Geograp 